\title{
Guidelines for model adaptation: a study of the transferability of a general seagrass ecosystem DBN model
}

\author{
Paula Hatum ${ }^{1}$, Kathryn McMahon ${ }^{2}$, Kerrie Mengersen ${ }^{1}$, and Paul $\mathrm{Wu}^{3}$ \\ ${ }^{1}$ Queensland University of Technology \\ ${ }^{2}$ Edith Cowan University \\ ${ }^{3}$ ARC Centre of Excellence in Mathematical and Statistical Frontiers
}

January 12, 2022

\begin{abstract}
Ecological models are extensively and increasingly used in support of environmental policy and decision making. Dynamic Bayesian Networks (DBN) as a tool for conservation have been demonstrated to be a valuable tool for providing a systematic and intuitive approach to integrating data and other critical information to help guide the decision-making process. However, data for a new ecosystem are often sparse. In this case, a general DBN developed for similar ecosystems could be applicable, but this may require the adaptation of key elements of the network. The research presented in this paper focused on a case study to identify and implement guidelines for model adaptation. We adapted a general DBN of a seagrass ecosystem to a new location where nodes were similar, but the conditional probability tables varied. We focused on two species of seagrass (Zostera noltei and Z. marina) located in Arcachon Bay, France. Expert knowledge was used to complement peer-reviewed literature to identify which components needed adjustment including parameterisation and quantification of the model and desired outcomes. We adopted both linguistic labels and scenario-based elicitation to elicit from experts the conditional probabilities used to quantify the DBN. Following the proposed guidelines, the model structure of the DBN was retained, but the conditional probability tables were adapted for nodes that characterised the growth dynamics in Zostera spp. population located in Arcachon Bay, as well as the seasonal variation on their reproduction. Particular attention was paid to the light variable as it is a crucial driver of growth and physiology for seagrasses. Our guidelines provide a way to adapt a general DBN to specific ecosystems to maximise model reuse and minimise re-development effort. Especially important from a transferability perspective are guidelines for ecosystems with limited data, and how simulation and prior predictive approaches can be used in these contexts.
\end{abstract}

\section{Hosted file}

Paper.pdf available at https://authorea.com/users/455146/articles/552529-guidelines-formodel-adaptation-a-study-of-the-transferability-of-a-general-seagrass-ecosystem-dbnmodel 

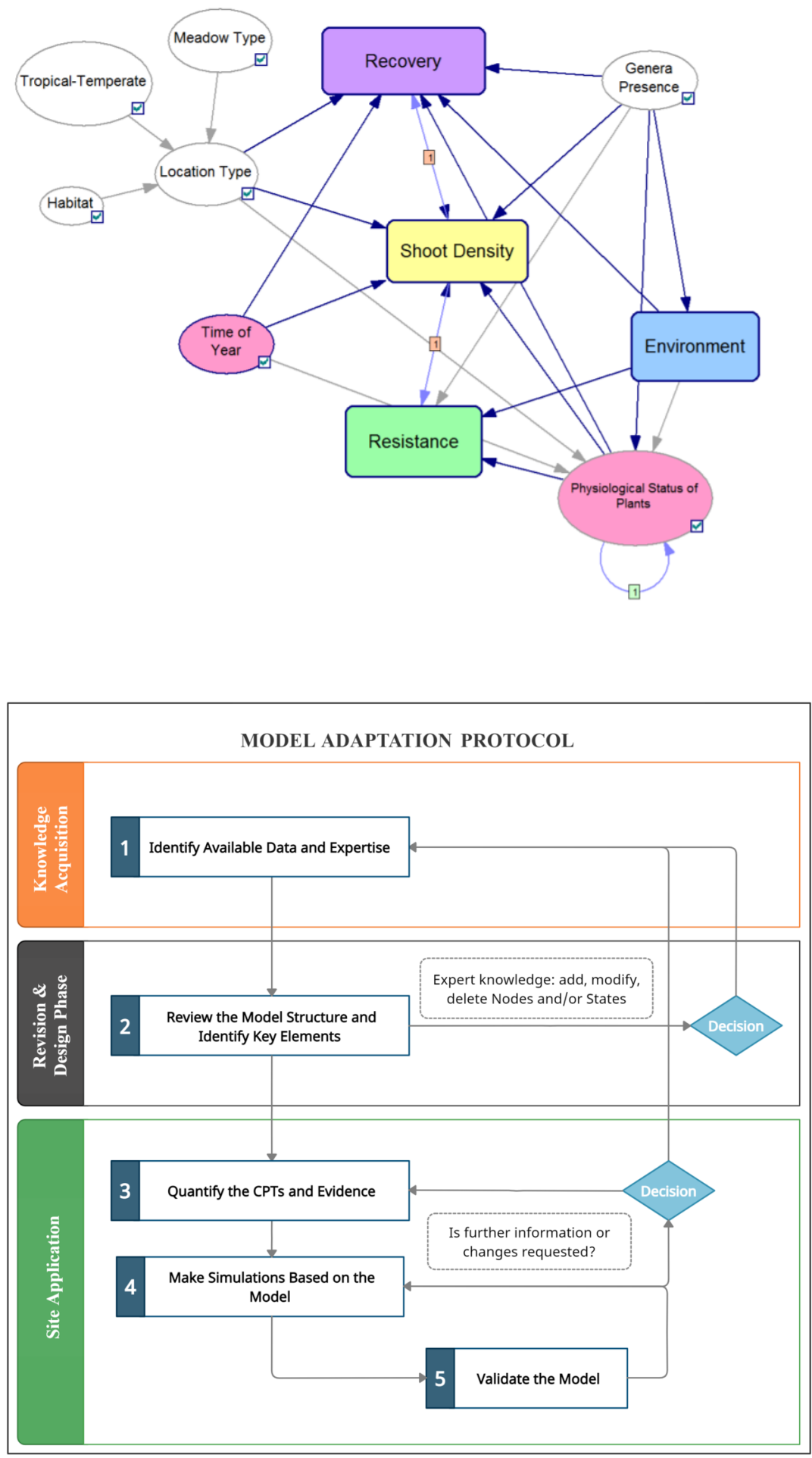


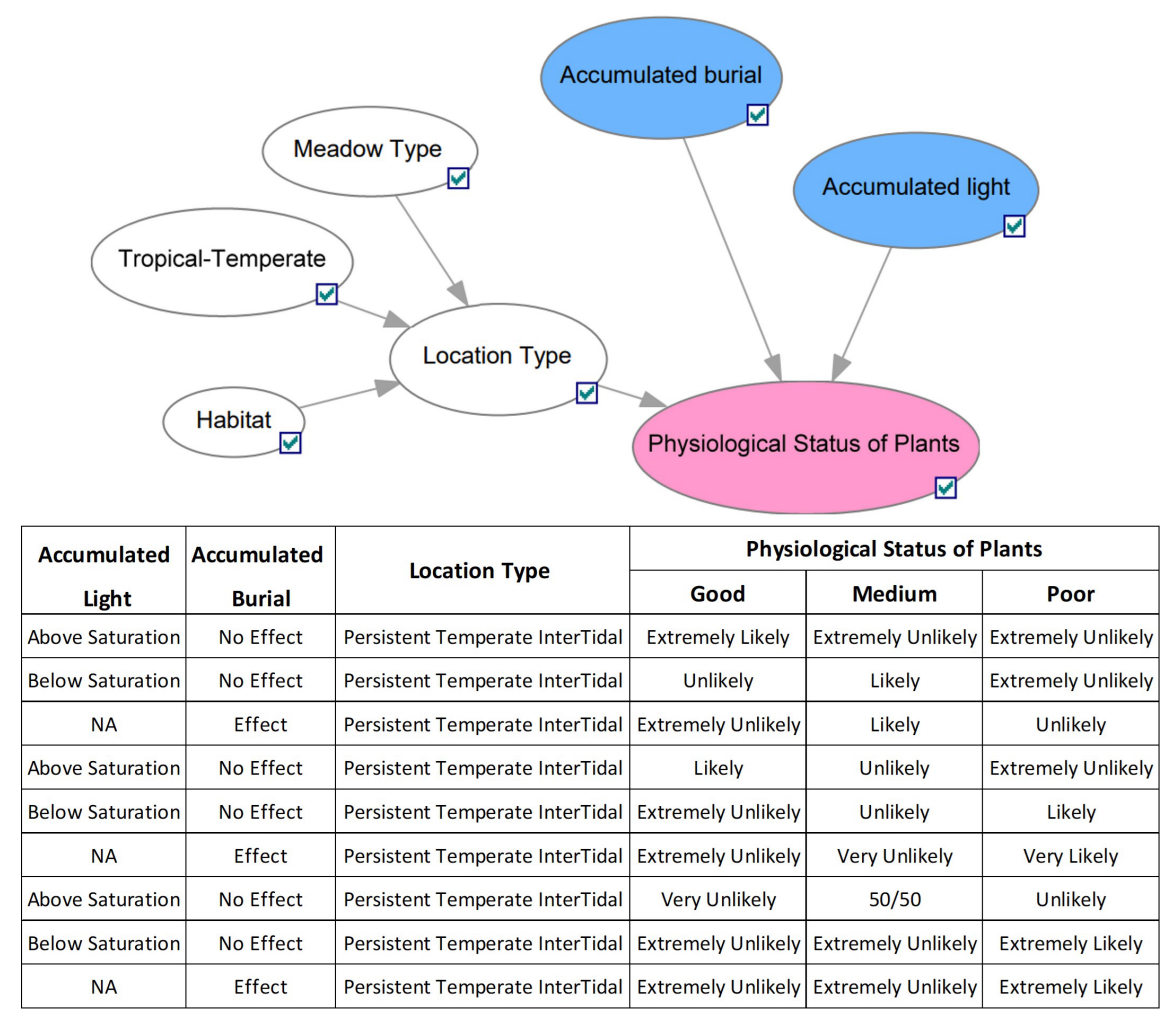


(a)

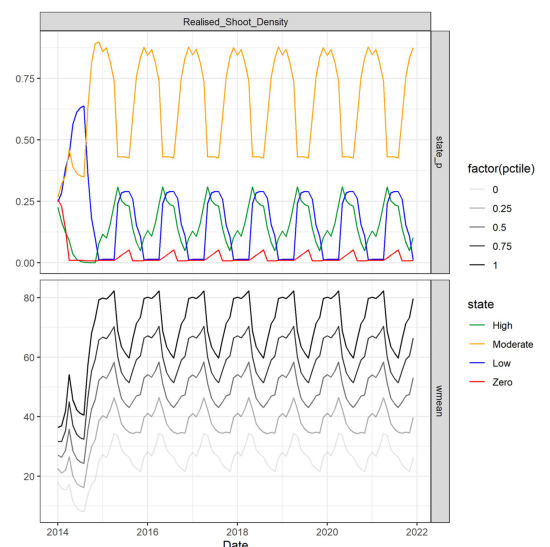

(c)

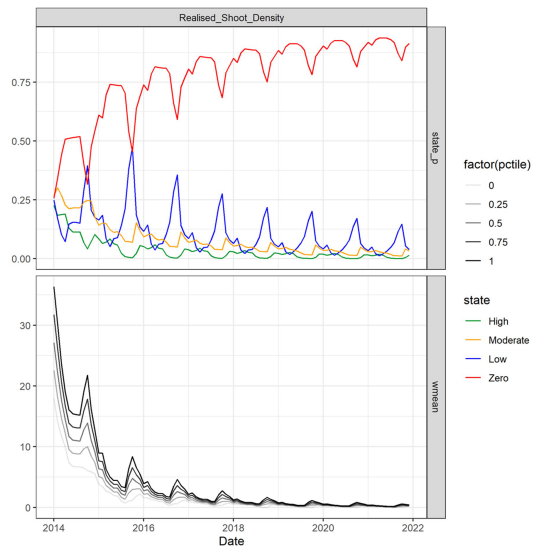

(b)

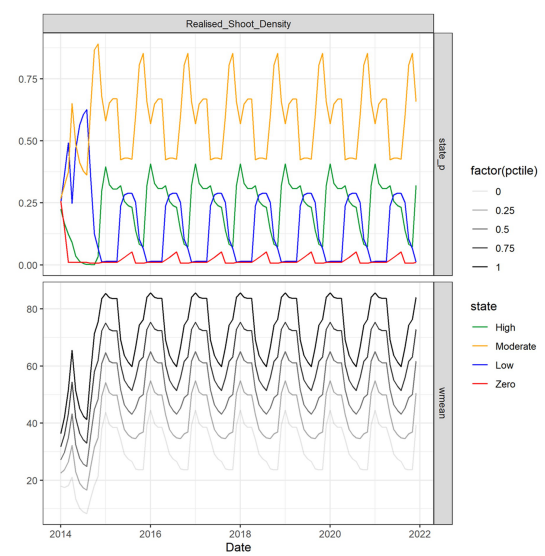

(d)

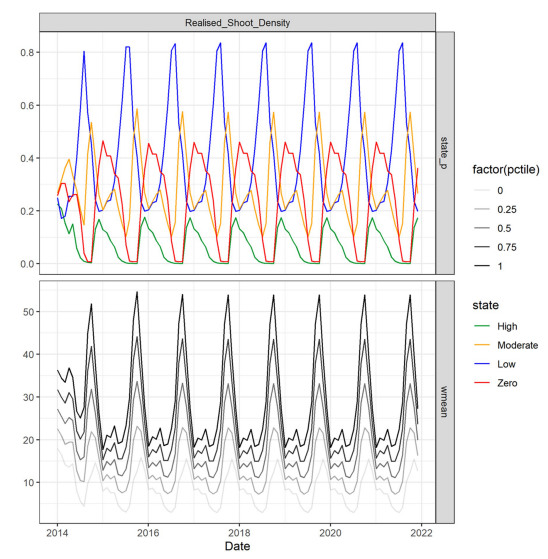

